\title{
ASSESSMENT OF THE IMPACTS OF INDUSTRIAL ACTIVITIES ON THE SOILS OF RECEIVING ENVIRONMENT AROUND SELECTED OPERATIONAL SITES IN IBADAN METROPOLIS, OYO STATE
}

\author{
Ogar P. A ${ }^{1}$, Ejiogu C. $C^{2}$, Uyo C. $\mathbf{N}^{2}$, Ukpe A. E $\mathbf{E}^{2}$, Esomonu $\mathrm{I}^{2}$ and Nwobodo F. $\mathrm{U}^{2}$ \\ ${ }^{1}$ Department of Geography, Obafemi Awolowo University, Ile-Ife, Nigeria. \\ ${ }^{2}$ Department of Environmental Management, Federal University of Technology, Owerri, \\ Nigeria. \\ Corresponding Author: Peter Ata Ogar
}

\begin{abstract}
The study examined the heavy metal components, physical and chemical parameters of soil samples around selected industrial establishments. This was to ascertain the level that industrial activities impacts on the soil quality with a view to providing a platform for rapid generation of data for environment related decision making and promoting compliance to environmental standards in Ibadan metropolis. Twelve composite soil samples to the depths of $0-15 \mathrm{~cm}, 15-30 \mathrm{~cm}, 30-45 \mathrm{~cm}$ to represent top and subsoil were collected at each of the selected industrial locations using soil auger for soil physical and chemical properties determination. Data collected were analysed using descriptive and inferential statistics. The mean $\mathrm{Pb}$ values for 0-15, 15-30, 30-45 cm soil depths were 5.66 $0.55,4.58 \pm 0.68,4.52 \pm 1.35 \mathrm{mg} / \mathrm{kg}$; Cd values were $10.23 \pm 0.60,9.40 \pm 1.33$ and $10.55 \pm 0.36 \mathrm{mg} / \mathrm{kg}$; Co values were $9.76 \pm 1.00,9.98 \pm 2.57$ and $13.58 \pm 4.16 \mathrm{mg} / \mathrm{kg}$; $\mathrm{Cr}$ were $10.18 \pm 2.40,12.53 \pm 3.17$ and $12.1 \pm 3.75$ and $\mathrm{Ni}$ were 11.17 $\pm 3.55,12.24 \pm 2.19$ and $12.53 \pm 2.34 \mathrm{mg} / \mathrm{kg}$. For Macro nutrients, Total Organic Carbon (TOC) mean values for BC was 33.50 $\pm 2.48,29.3 \pm 3.91,26.6 \pm 2.74$ and $3.60 \pm 0.16$ and TN values were $3.60 \pm 0.16,3.40 \pm 0.12$, and $2.90 \pm 0.28$ respectively while AC had Total Organic Carbon mean values of $28.03 \pm 0.65,23.5 \pm 2.01,19.24 \pm 1.43$ and Total Nitrogen (TN) mean values $2.90 \pm 0.07,2.43 \pm 0.21$ and $1.99 \pm 0.15$ respectively. For Micro nutrient $\mathrm{Mn}, \mathrm{Fe}, \mathrm{Cu}$, $\mathrm{Zn}$, the mean values at 0-15, 15-30 and 30-45 soil depth for BC were $39.08 \pm 9.57,32.43 \pm$ 11.93 and $18.78 \pm 4.80$ for $M n$, mean values for Fe were $39.53 \pm 15.58,51.83 \pm 14.62$ and $34.33 \pm 10.68$, also mean values for $\mathrm{Cu}$ was $8.53 \pm 0.32,8.26 \pm 0.29,7.99 \pm 0.06$ and mean values for Zn were $9.01 \pm 0.66,6.62 \pm 0.21$ and $4.42 \pm 1.11$ respectively. There was a decrease in value with depth which may be associated with downward movement of organic materials and nutrients. The study concluded that industrial activities are impacting on the soil quality with special reference to nutrient and heavy metal contents within and around industrial facilities.
\end{abstract}

Key words: Industrial Activities, Soil Characteristics, Heavy Metals, Industrial pollution 


\section{Introduction}

Industrial activities result in heavy metals and toxic components that accumulate in soils. Metal and toxic contaminated soils can cause problems to animals and plants as well as human beings when they consume such plants and animals (Tiller, 1986). Heavy metals can accumulate in soil when industrial effluents are used for irrigation purposes or when not properly disposed (EL-Nennah et al., 2006). Heavy metal pollution in soils arises from various anthropogenic activities such as industrial operations that releas treated and untreated sewage water and sewage sludge on soils (EL Handy, 2007, Aghabarati, 2008, Aiyesami, 2005, Liu and Richard, 2010, Adams 2010, Adedeji et al, 2011)

Environmental pollution from industries in many major urban centres in Nigeria such as Ibadan has increased noticeably and has continued to have adverse effect on the immediate and distant environment of the industries (receiving ecosystems) (Osibanjo et al., 2011, Tijani and Onodera, 2004). The impacts include physical landscape changes/disturbance, surface and ground water pollution, air pollution, noise, soil erosion, sedimentation, destruction of biodiversity, destruction of water biota, and land degradation (Tijani et al., 2004, Awomeso et al., 2009, Ogedengbe and Akinbile, 2010).

Most pollution of soil resources are observed at surrounding areas of the industries (Adeboye, 2002). Resistance and stability of the heavy elements in soil is very long than other pollutants and soil pollution by the heavy metals is relatively permanent. The heavy metals include lead, cadmium, silver and mercury which their detrimental effects have been proven on the living creatures and have repeatedly caused environmental incidents (Lin, 2010, Ogunyemi, 2003, Tiller, 1986). Some of these detrimental effects of the heavy metals include as follows: disorder of biological activities of the soil, toxic effects on plants and harmful effects on human being as a result of entrance of materials to the food chain (Adedipe et al, 2005, Adeoye, 2008).

The study area which was Ibadan consisted of 11 Local Government Areas (LGAs) for governance and administrative purposes. Five of the LGAs are located in the metropolis, while the remaining six are either predominantly peri-urban or rural settlements. Ibadan has a total land area of $3123 \mathrm{~km}$, of which about 15 percent is urban and the remaining 85 percent is classified as peri-urban (Adelekan et al., 2014). Ibadan North LGA is the largest among the urban LGAs $\left(145.58 \mathrm{~km}^{2}\right.$ ) while Ibadan North West is the smallest at $31.38 \mathrm{~km}^{2}$. The periurban LGA of Ido $\left(865.49 \mathrm{~km}^{2}\right)$ covers the largest land area (Adelekan et al., 2014).

The soils of Ibadan region were formed from rocks of the Pre-cambrain basement complex formation, especially, granites gneisses, guartz-schist, biotite gneisses and schists. They were formed under moist semi-deciduous forest cover (Brady, 2009) and belong to the major soil group called Ferruginous tropical soils (Aweto, 1994). The soils have been mapped and classified into soil associations and series by Smyth and Montgomery (1962). Four soil associations occur in Ibadan region. They are: (i) Iwo, (ii) Okemesi, (iii) Egbeda and (iv) Mamu soil associations. The classification is largely based on soil parent materials.

\section{Materials and Method}

The selected industrial facilities were divided into five points namely Southern, Northern, Eastern, Western portions and Central (middle) points. Three sampling depths were taken at the selected industrial locations using soil auger, namely; $0-15 \mathrm{~cm}, 15-30 \mathrm{~cm}, 30-45$ $\mathrm{cm}$ to represent the top and sub soils. Soil samples from the related axis of the factory were bulked to produce a composite. The soil samples were taken to the laboratory, air dried and 
sieved through two millimeter sieve for soil chemical and physical analysis. The soil total nitrogen (TN) and total organic carbon (TOC) were determined by kjeldahl method. Soil pH was determined using 1:2 soil water suspension. Particle size was determined by the hydrometer method. Available $\mathrm{P}$ was determined by Bray P1. Exchangeable K, sodium (Na), calcium (Ca) and magnesium $(\mathrm{Mg})$ were first extracted using neutral normal ammonium acetate $\left(\mathrm{NH}_{4} \mathrm{OAC}\right)$. Thereafter, $\mathrm{K}$ and $\mathrm{Na}$ were determined by flame emission while $\mathrm{Mg}$ and $\mathrm{Ca}$ were determined by atomic absorption. Soil samples were digested in Teflon beakers with a mixture of $\mathrm{HNO}_{3}, \mathrm{HCl}$ and HF. Similarly, Blanks were prepared to check for background contamination by the reagents used. The digested samples were analyzed for heavy metals using Atomic Absorption Spectrophotometer (AAS). Descriptive and inferential statistic was used to determine the mean values, range and standard errors of each soil property.

\section{Results and Discussion}

The chemical properties and means of the soils of the study area comprising $\mathrm{pH}$, Total organic carbon, Total Nitrogen, Available phosphorous, exchangeable acidity, extractable alkalinity, exchangeable bases, micro nutrient and their mean are presented on Tables 1,2,7 and 8 below. The heavy metal distribution and their mean are presented in Tables 3 and 9, while the particle size distribution and textural classes of the soil with their means are presented in Tables 6 and 12 , that is mean values of heavy metals at various depth $0-15 \mathrm{~cm}, 15-30 \mathrm{~cm}$ and $30-45 \mathrm{~cm}$ soil depths. The acidity of the soil is a measure of the Cation Exchange Capacity (CEC) occupied by $\mathrm{H}+$ and $\mathrm{Al}_{3}+$, mean $\mathrm{pH}$ values of $\mathrm{AC}$ at depth $0-15,15-30$ and $30-45 \mathrm{~cm}$ were $6.25 \pm 0.50,6.28$ $\pm 0.08,6.4 \pm 0.05$ respectively while mean values for $\mathrm{BC}$ were $6.63 \pm 1.80,6.77 \pm 0.048$ and $6.80 \pm 0.17$ respectively indicating that the soils were slightly acidic. The more acidic the soil is, the lower the value of $\mathrm{pH}$ and as soil acidity increases soluble aluminium and manganese increase to toxic levels (Agdex, 2010). Macro nutrients, Total Organic Carbon (TOC) mean values for $\mathrm{BC}$ were $33.50 \pm 2.48,29.3 \pm 3.91,26.6 \pm 2.74$ and $3.60 \pm 0.16$ and Total Nitrogen (TN) values were $3.60 \pm 0.16,3.40 \pm 0.12$, and $2.90 \pm 0.28$ respectively while AC had Total Organic Carbon (TOC) mean values of $28.03 \pm 0.65,23.5 \pm 2.01,19.24 \pm 1.43$ and Total Nitrogen mean $(\mathrm{TN})$ values $2.90 \pm 0.07,2.43 \pm 0.21$ and $1.99 \pm 0.15$ respectively. Micro nutrient worked on were $\mathrm{Mn}, \mathrm{Fe}, \mathrm{Cu}, \mathrm{Zn}$, the mean values at $0-15,15-30$ and 30-45 soil depth for $\mathrm{AC}$ were $39.08 \pm 9.57,32.43 \pm 11.93$ and $18.78 \pm 4.80$ for $\mathrm{Mn}$, mean values for Fe were $39.53 \pm 15.58,51.83 \pm 14.62$ and $34.33 \pm 10.68$, also mean values for $\mathrm{Cu}$ were $8.53 \pm 0.32$, $8.26 \pm 0.29,7.99 \pm 0.06$ and mean values for $\mathrm{Zn}$ were $9.01 \pm 0.66,6.62 \pm 0.21$ and $4.42 \pm 1.11$ respectively. Like $\mathrm{pH}$ there was a decrease in value with depth which may be associated with downward movement of organic materials and nutrients. $\mathrm{Mn}, \mathrm{Fe}, \mathrm{Cu}$, and $\mathrm{Zn}$ showed regular decrease values were high for plant (Zia et al, 2006) but below contamination levels of soil (Thorton et al., 2006). Heavy metals reported in plants may mainly come from the soil in which they were grown (Lin et al, 2010). Heavy metals analyzed were $\mathrm{Pb}, \mathrm{Cd}, \mathrm{Co}, \mathrm{Cr}$ and $\mathrm{Ni}$. Mean values recorded for $\mathrm{Pb}$ at $\mathrm{AC}$ were $66 \pm 0.55,4.58 \pm 0.68,4.52 \pm 1.35, \mathrm{Cd}$ was $10.23 \pm 0.63,9.4$ \pm 1.33 and $10.55 \pm 0.36$, Co was $9.76 \pm 1.00,9.98 \pm 2.59,13.58 \pm 4.16$ and $12.1 \pm 3.75$ while $\mathrm{Ni}$ had values of $11.17 \pm 3.55,12.34 \pm 2.9$ and $12.53 \pm 2.34$ respectively at different soil depths. Mean of heavy metal distribution of the soil at $\mathrm{BC}$ were $\mathrm{Pb}: 1.62 \pm 0.72,1.25 \pm 0.69$ and $1.44 \pm$ $0.76, \mathrm{Cd}$ values were $1.12 \pm 0.60,1.24 \pm 0.71$ and $1.29 \pm 0.73$, Co was $1.43 \pm 0.73,1.44 \pm 0.76$ and $1.42 \pm 0.77$, Cr value was $3.00 \pm 1.00,3.09 \pm 1.17$ and $3.20 \pm 1.10$ while Ni had values of $5.13 \pm 1.17,3.09 \pm 0.75$ and $4.05 \pm 1.82$ respectively at stated soil depths. The mean values for heavy metals were higher than the first normal level but has not reached critical contamination levels. The range of soils particle size distribution was as follows; clay $113 \pm 12.58-183 \pm$ $51.23 \mathrm{~g} / \mathrm{kg}, 165 \pm 15.00-215 \pm 33.04 \mathrm{~g} / \mathrm{kg}$ for silt and $647 \pm 50.58-662 \pm 35.12$ for sand of 
AC while BC had values $106 \pm 13.50-172 \pm 54.37 \mathrm{~g} / \mathrm{kg}$ for clay, $155 \pm 15.55-208 \pm 28.69$ $\mathrm{g} / \mathrm{kg}$ silt and $574 \pm 50.23-609 \pm 21.36 \mathrm{~g} / \mathrm{kg}$ sand. Textural classes ranged from sandy loam to sandy clay loam suggesting a sandy soil which was vulnerable to leaching especially of toxic chemicals. 
Journal DOI: www.doi.org/10.46654/ij.24889849 Vol. 7, Issue 2 (February, 2021) |www.ijaar.org

Article DOI: www.doi.org/10.46654/ij.24889849.e7218

\section{Table 1: $\quad$ Soil Chemical Properties at BC, Ibadan}

\begin{tabular}{|c|c|c|c|c|c|c|c|c|c|c|c|c|c|c|c|}
\hline Location & $\begin{array}{l}\text { Depth } \\
(\mathrm{cm})\end{array}$ & $\mathrm{pH}$ & $\begin{array}{l}\text { TOC } \\
(\mathrm{g} / \mathrm{kg})\end{array}$ & $\begin{array}{l}\text { TN } \\
(\mathrm{g} / \mathrm{kg})\end{array}$ & $\begin{array}{l}\text { AVP } \\
(\mathrm{mg} / \mathrm{kg})\end{array}$ & $\begin{array}{l}\text { Ex. } \\
\text { Acidity } \\
(\mathrm{mg} / \mathrm{l})\end{array}$ & $\begin{array}{l}\text { Ext. } \\
\text { Alkalinity } \\
(\mathrm{mg} / \mathrm{l})\end{array}$ & $\begin{array}{l}\mathrm{Mn} \\
(\mathrm{mg} / \mathrm{l})\end{array}$ & $\begin{array}{l}\mathrm{Fe} \\
(\mathrm{mg} / \mathrm{kg})\end{array}$ & $\begin{array}{l}\mathrm{Cu} \\
(\mathrm{mg} / \mathrm{kg})\end{array}$ & $\begin{array}{l}\mathrm{Zn} \\
(\mathrm{mg} / \mathrm{l})\end{array}$ & $\begin{array}{l}\mathrm{K} \\
(\mathrm{mg} / \mathrm{l})\end{array}$ & $\begin{array}{l}\mathrm{Ca} \\
(\mathrm{cmol} / \mathrm{kg})\end{array}$ & $\begin{array}{l}\mathrm{Mg} \\
(\mathrm{cmol} / \mathrm{l})\end{array}$ & $\begin{array}{l}\mathrm{Na} \\
(\mathrm{cmol} / \mathrm{kg})\end{array}$ \\
\hline & $0-15$ & 6.9 & 30.04 & 3.52 & 39.10 & 0.6 & 1.3 & 9.4 & 9.2 & 6.9 & 4.51 & 0.60 & 8.62 & 1.24 & 2.22 \\
\hline \multirow[t]{3}{*}{ ETP A } & $15-30$ & 6.7 & 29.26 & 3.30 & 37.12 & 0.3 & 1.1 & 3.8 & 7.6 & 6.20 & 4.30 & 0.24 & 6.30 & 1.00 & 1.90 \\
\hline & $30-45$ & 6.3 & 24.13 & 2.14 & 37.10 & 0.2 & 1.2 & 3.5 & 6.4 & 5.21 & 4.12 & 0.22 & 6.19 & 1.00 & 2.98 \\
\hline & $0-15$ & 6.7 & 34.26 & 3.89 & 37.30 & 0.2 & 1.8 & 8.0 & 7.1 & 5.22 & 4.11 & 1.18 & 5.90 & 0.64 & 3.00 \\
\hline \multirow[t]{2}{*}{ Central } & $15-30$ & 6.7 & 20.22 & 3.42 & 36.75 & 0.1 & 1.4 & 20.2 & 6.2 & 4.12 & 3.80 & 1.05 & 4.70 & 0.53 & 2.87 \\
\hline & $0-15$ & 6.8 & 29.47 & 3.20 & 38.62 & 0.5 & 1.4 & 20.2 & 8.2 & 3.31 & 2.20 & 0.31 & 7.54 & 0.22 & 2.99 \\
\hline Western & $15-30$ & 6.8 & 28.36 & 3.15 & 37.32 & 0.1 & 1.4 & 15.2 & 6.1 & 2.50 & 1.21 & 0.15 & 6.42 & 0.20 & 2.00 \\
\hline fenceline & $30-45$ & 6.9 & 29.52 & 3.12 & 36.63 & 0.1 & 1.2 & 18.8 & 4.9 & 2.30 & 1.20 & 0.12 & 6.30 & 0.12 & 2.21 \\
\hline Eastern & $0-15$ & 6.1 & 40.23 & 3.80 & 37.38 & 0.1 & 1.1 & 28.0 & 9.1 & 2.54 & 1.30 & 1.26 & 8.12 & 0.22 & 2.62 \\
\hline \multirow[t]{2}{*}{ Fenceline } & $15-30$ & 6.9 & 39.31 & 3.73 & 37.22 & 0.1 & 1.5 & 20 & 8.9 & 1.82 & 1.22 & 0.40 & 7.86 & 0.20 & 2.14 \\
\hline & $30-45$ & 7.1 & 32.42 & 3.45 & 36.46 & 0.1 & 1.0 & 18 & 8.6 & 1.21 & 1.10 & 0.25 & 6.74 & 0.20 & 2.81 \\
\hline
\end{tabular}

N:B ETP A= Effluent Treatment Plant Axis; Ex. Acidity= Exchangeable acidity, Ext. Alkalinity = Extractable alkalinity, TN = Total Nitrogen, $\mathrm{Mn}, \mathrm{Fe}, \mathrm{Cu}, \mathrm{Zn}(\mathrm{mg} / \mathrm{kg}), \mathrm{Ca}, \mathrm{K}, \mathrm{Mg}, \mathrm{Na}(\mathrm{cmol} / \mathrm{kg})$., TOC = Total Organic Carbon. 
International Journal of Advanced Academic Research (Sciences, Technology and Engineering) | ISSN: 2488-9849

Journal DOI: www.doi.org/10.46654/ij.24889849

Vol. 7, Issue 2 (February, 2021) |www.ijaar.org

Article DOI: $\underline{\text { ww.doi.org/10.46654/ij.24889849.e7218 }}$

Table 2: $\quad$ Mean of Soil Chemical Properties at BC, Ibadan

\begin{tabular}{|c|c|c|c|c|c|c|c|c|c|c|c|c|c|c|}
\hline $\begin{array}{l}\text { Depth } \\
\text { (cm) }\end{array}$ & $\mathrm{pH}$ & $\begin{array}{l}\text { TOC } \\
(\mathrm{g} / \mathrm{kg})\end{array}$ & $\begin{array}{l}\text { TN } \\
(\mathrm{g} / \mathrm{kg})\end{array}$ & $\begin{array}{l}\text { AVP } \\
(\mathrm{mg} / \mathrm{kg})\end{array}$ & $\begin{array}{l}\text { Ex. } \\
\text { Acidity } \\
(\mathrm{mg} / \mathrm{l})\end{array}$ & $\begin{array}{l}\text { Ext. } \\
\text { Alkalinity } \\
(\mathrm{mg} / \mathrm{l})\end{array}$ & $\begin{array}{l}\mathrm{Mn} \\
(\mathrm{mg} / \mathrm{l})\end{array}$ & $\begin{array}{l}\mathrm{Fe} \\
(\mathrm{mg} / \mathrm{kg})\end{array}$ & $\begin{array}{l}\mathrm{Cu} \\
(\mathrm{mg} / \mathrm{kg})\end{array}$ & $\begin{array}{l}\mathrm{Zn} \\
(\mathrm{mg} / \mathrm{l})\end{array}$ & $\begin{array}{l}\mathrm{K} \\
(\mathrm{mg} / \mathrm{l})\end{array}$ & $\begin{array}{l}\mathrm{Ca} \\
(\mathrm{cmol} / \mathrm{kg})\end{array}$ & $\begin{array}{l}\mathrm{Mg} \\
(\mathrm{cmol} / \mathrm{l})\end{array}$ & $\begin{array}{l}\mathrm{Na} \\
(\mathrm{cmol} / \mathrm{kg})\end{array}$ \\
\hline $0-15$ & $6.63 \pm 0.180$ & $33.50 \pm 2.48$ & $3.60 \pm 0.16$ & $38.09 \pm 0.44$ & $0.35 \pm 0.12$ & $1.40 \pm 0.15$ & $16.40 \pm 4.73$ & $8.40 \pm 0.49$ & $4.59 \pm 0.98$ & $3.03 \pm 0.77$ & $0.83 \pm 0.23$ & $7.54 \pm 0.59$ & $0.58 \pm 0.24$ & $2.71 \pm 0.18$ \\
\hline $15-30$ & $6.77 \pm 0.048$ & $29.3 \pm 3.91$ & $3.40 \pm 0.12$ & $37.10 \pm 0.12$ & $0.15 \pm 0.05$ & $1.20 \pm 0.09$ & $14.80 \pm 3.84$ & $7.20 \pm 0.66$ & $3.66 \pm 0.97$ & $2.63 \pm 0.82$ & $0.46 \pm 0.20$ & $6.32 \pm 0.65$ & $0.48 \pm 0.19$ & $2.22 \pm 0.22$ \\
\hline $30-45$ & $6.80 \pm 0.17$ & $26.6 \pm 2.74$ & $2.90 \pm 0.28$ & $36.69 \pm 0.14$ & $0.12 \pm 0.03$ & $1.12 \pm 0.05$ & $13.60 \pm 3.52$ & $6.30 \pm 0.84$ & $3.21 \pm 0.90$ & $2.35 \pm 0.73$ & $0.20 \pm 0.03$ & $5.80 \pm 0.57$ & $0.55 \pm 0.23$ & $2.44 \pm 0.28$ \\
\hline
\end{tabular}

Source: Fieldwork 
Journal DOI: www.doi.org/10.46654/ij.24889849

Vol. 7, Issue 2 (February, 2021) |www.ijaar.org

Article DOI: $\underline{\text { ww.doi.org/10.46654/ij.24889849.e7218 }}$

Table 3: Heavy Metal Distribution of the soils at BC, Ibadan

\begin{tabular}{|c|c|c|c|c|c|c|}
\hline Location & Depth (cm) & $\mathrm{Pb}$ (mg/kg) & Cd (mg/kg) & Co (mg/kg) & $\mathrm{Cr}$ (mg/kg) & $\mathrm{Ni}(\mathrm{mg} / \mathrm{kg})$ \\
\hline & $0-15$ & 2.80 & 2.18 & 2.89 & 5.22 & 8.01 \\
\hline \multirow[t]{3}{*}{ ETP A } & $15-30$ & 2.70 & 2.12 & 2.76 & 4.21 & 5.02 \\
\hline & $30-45$ & 1.90 & 2.00 & 2.50 & 3.76 & 4.34 \\
\hline & $0-15$ & 2.90 & 2.90 & 2.88 & 5.10 & 8.32 \\
\hline \multirow[t]{3}{*}{ Central } & $15-30$ & 2.90 & 2.87 & 2.76 & 4.90 & 3.56 \\
\hline & $30-45$ & 2.80 & 2.22 & 2.62 & 6.20 & 9.10 \\
\hline & $0-15$ & 0.66 & 0.13 & 0.25 & 0.80 & 2.21 \\
\hline \multirow[t]{2}{*}{ Western fenceline } & $15-30$ & 0.15 & 0.01 & 0.15 & 0.45 & 1.90 \\
\hline & $30-45$ & 0.10 & 0.01 & 0.13 & 1.70 & 1.89 \\
\hline Eastern & $0-15$ & 0.12 & 0.10 & 0.10 & 1.90 & 2.00 \\
\hline \multirow[t]{2}{*}{ Fenceline } & $15-30$ & 0.10 & 0.10 & 0.10 & 1.82 & 1.90 \\
\hline & $30-45$ & 0.10 & 0.04 & 0.06 & 1.44 & 0.90 \\
\hline
\end{tabular}

Table 4: $\quad$ Mean of Heavy Metal Distribution of the soils at BC, Ibadan

\begin{tabular}{llllr}
\hline Depth (cm) & Pb (mg/kg) & Cd (mg/kg) & Co (mg/kg) & Cr (mg/kg) \\
\hline $0-15$ & $1.62 \pm 0.72$ & $1.12 \pm 0.60$ & $1.43 \pm 0.73$ & $3.00 \pm 1.00$ \\
$15-30$ & $1.25 \pm 0.69$ & $1.24 \pm 0.71$ & $1.44 \pm 0.76$ & $3.09 \pm 1.17$ \\
$30-45$ & $1.44 \pm 0.76$ & $1.29 \pm 0.73$ & $1.42 \pm 0.77$ & $3.20 \pm 1.10$ \\
\hline
\end{tabular}


Journal DOI: www.doi.org/10.46654/ij.24889849

Vol. 7, Issue 2 (February, 2021) |www.ijaar.org

Article DOI: $\underline{\text { ww.doi.org/10.46654/ij.24889849.e7218 }}$

Table 5: $\quad$ Physical Properties of the Soils at BC, Ibadan

\begin{tabular}{|c|c|c|c|c|c|}
\hline Location & Depth (cm) & Clay (g/kg) & Silt (g/kg) & Sand (g/kg) & Textural Class \\
\hline & $0-15$ & 90 & 260 & 496 & Sandy Loam \\
\hline \multirow[t]{3}{*}{ ETP A } & $15-30$ & 124 & 170 & 596 & Sandy Loam \\
\hline & $30-45$ & 94 & 120 & 646 & Sandy Loam \\
\hline & $0-15$ & 104 & 140 & 716 & Sandy Loam \\
\hline \multirow[t]{3}{*}{ Central } & $15-30$ & 204 & 180 & 656 & Sandy Clay Loam \\
\hline & $30-45$ & 244 & 140 & 596 & Sandy Clay Loam \\
\hline & $0-15$ & 144 & 250 & 596 & Loamy Sand \\
\hline \multirow[t]{2}{*}{ Western fenceline } & $15-30$ & 264 & 170 & 556 & Loamy Sand \\
\hline & $30-45$ & 284 & 170 & 426 & Loamy Sand \\
\hline Eastern & $0-15$ & 84 & 180 & 616 & Sandy Clay Loam \\
\hline \multirow[t]{2}{*}{ Fenceline } & $15-30$ & 64 & 190 & 626 & Sandy Clay Loam \\
\hline & $30-45$ & 64 & 190 & 626 & Sandy Clay Loam \\
\hline
\end{tabular}

Table 6: Mean of Soil Particle Size Distribution at BC, Ibadan

\begin{tabular}{llll}
\hline Depth (cm) & Clay $(\mathbf{g} / \mathbf{k g})$ & Silt (g/kg) & Sand (g/kg) \\
\hline $0-15$ & $106 \pm 13.50$ & $208 \pm 28.69$ & $606 \pm 45.09$ \\
$15-30$ & $164 \pm 43.97$ & $178 \pm 4.79$ & $609 \pm 21.36$ \\
$30-45$ & $172 \pm 54.37$ & $155 \pm 15.55$ & $574 \pm 50.23$ \\
\hline
\end{tabular}


Journal DOI: www.doi.org/10.46654/ij.24889849 Vol. 7, Issue 2 (February, 2021) | www.ijaar.org

Article DOI: $\underline{\text { ww.doi.org/10.46654/ij.24889849.e7218 }}$

Table 7: $\quad$ Soil Chemical Properties at AC, Ibadan

\begin{tabular}{|c|c|c|c|c|c|c|c|c|c|c|c|c|c|c|c|}
\hline Location & $\begin{array}{l}\text { Depth } \\
\text { (cm) }\end{array}$ & pH & $\begin{array}{l}\text { TOC } \\
\text { (g/kg) }\end{array}$ & $\begin{array}{l}\text { TN } \\
\text { (g/kg) }\end{array}$ & $\begin{array}{l}\text { AVP } \\
(\mathrm{mg} / \mathbf{k g})\end{array}$ & $\begin{array}{l}\text { Ex. } \\
\text { Acidity } \\
\text { (mg/kg) }\end{array}$ & $\begin{array}{l}\text { Ext. } \\
\text { Alkalinity } \\
\text { (mg/kg) }\end{array}$ & $\begin{array}{l}\text { Mn } \\
(\mathrm{mg} / \mathrm{kg})\end{array}$ & $\begin{array}{l}\text { Fe } \\
(\mathrm{mg} / \mathrm{kg})\end{array}$ & $\begin{array}{l}\text { Cu } \\
(\mathrm{mg} / \mathrm{kg})\end{array}$ & $\begin{array}{l}\text { Zn } \\
\text { (mg/kg) }\end{array}$ & $\begin{array}{l}\text { K } \\
(\mathrm{mg} / \mathrm{kg})\end{array}$ & $\begin{array}{l}\text { Ca } \\
(\mathrm{cmol} / \mathrm{kg})\end{array}$ & $\begin{array}{l}\text { Mg } \\
\text { (cmol/kg) }\end{array}$ & $\begin{array}{l}\text { Na } \\
\text { (cmol/kg) }\end{array}$ \\
\hline & $0-15$ & 6.3 & 26.64 & 2.76 & 37.34 & 0.1 & 1.9 & 11.2 & 60.7 & 8.63 & 5.24 & 0.2 & 11.85 & 1.83 & 3.09 \\
\hline \multirow[t]{3}{*}{ Pit } & $15-30$ & 6.2 & 21.46 & 2.22 & 36.66 & 0.1 & 1.4 & 4.9 & 32.3 & 7.52 & 3.77 & 0.14 & 8.26 & 1.14 & 3.09 \\
\hline & $30-45$ & 6.3 & 16.28 & 1.68 & 35.98 & 0.1 & 1.3 & 4.7 & 31.9 & 8.02 & 3.31 & 0.17 & 9.28 & 1.22 & 3.13 \\
\hline & $0-15$ & 6.1 & 29.6 & 3.06 & 35.83 & 0.2 & 1.6 & 54.8 & 70.2 & 8.72 & 8.23 & 0.34 & 10.2 & 0.91 & 2.96 \\
\hline \multirow[t]{3}{*}{ Central } & $15-30$ & 6.1 & 27.38 & 2.83 & 34.48 & 0.1 & 1 & 62.7 & 89.9 & 8.73 & 5.44 & 2.05 & 11.22 & 0.47 & 2.87 \\
\hline & $30-45$ & 6.4 & 21.09 & 2.18 & 33.33 & 0.4 & 1.3 & 22.1 & 59.5 & 8.06 & 7.75 & 0.23 & 13.9 & 0.63 & 2.91 \\
\hline & $0-15$ & 6.3 & 27.38 & 2.83 & 34.01 & 0.6 & 1.5 & 45.4 & 23.1 & 9.14 & 6.19 & 3.94 & 10.6 & 0.66 & 2.7 \\
\hline \multirow[t]{3}{*}{$\mathrm{E} / \mathrm{W}$} & $15-30$ & 6.4 & 18.87 & 1.95 & 34.01 & 0.2 & 1.4 & 27.1 & 25.8 & 8.05 & 3.76 & 0.24 & 10.53 & 0.45 & 3.13 \\
\hline & $30-45$ & 6.5 & 17.39 & 1.8 & 34.78 & 0.1 & 1.1 & 26.3 & 38.3 & 7.81 & 3.12 & 0.22 & 10.63 & 0.74 & 2.83 \\
\hline & $0-15$ & 6.3 & 28.49 & 2.95 & 41.24 & 0.1 & 1.1 & 44.9 & 4.1 & 7.64 & 16.37 & 0,47 & 11.43 & 0.58 & 2.83 \\
\hline \multirow[t]{2}{*}{$\mathrm{N} / \mathrm{S}$} & $15-30$ & 6.4 & 26.27 & 2.75 & 36.56 & 0.2 & 1 & 35 & 59.3 & 8.72 & 13.51 & 0.21 & 13.37 & 0.69 & 2.74 \\
\hline & $30-45$ & 6.5 & 22.2 & 2.3 & 36.14 & 0.2 & 0.8 & 22 & 7.6 & 8.06 & 3.51 & 0.2 & 7.86 & 0.64 & 2.96 \\
\hline
\end{tabular}

N.B E/W= East/West; Ex. Acidity = Exchangeable acidity, N/S= North/South, Ext. Alkalinity = Extractable alkalinity, TN = Total Nitrogen,

$\mathrm{Mn}, \mathrm{Fe}, \mathrm{Cu}, \mathrm{Zn}(\mathrm{mg} / \mathrm{kg}), \mathrm{Ca}, \mathrm{K}, \mathrm{Mg}, \mathrm{Na}(\mathrm{cmol} / \mathrm{kg})$., TOC = Total Organic Carbon. 
International Journal of Advanced Academic Research (Sciences, Technology and Engineering) | ISSN: 2488-9849

Journal DOI: www.doi.org/10.46654/ij.24889849

Vol. 7, Issue 2 (February, 2021) | www.ijaar.org

Article DOI: www.doi.org/10.46654/ij.24889849.e7218

Table 8: $\quad$ Mean of Soil Chemical Properties at AC, Ibadan

\begin{tabular}{|c|c|c|c|c|c|c|c|c|c|c|c|c|c|c|}
\hline $\begin{array}{l}\text { Depth } \\
\text { (cm) }\end{array}$ & pH & $\begin{array}{l}\text { TOC } \\
(\mathrm{g} / \mathrm{kg})\end{array}$ & $\begin{array}{l}\text { TN } \\
(\mathrm{g} / \mathrm{kg})\end{array}$ & $\begin{array}{l}\text { AVP } \\
(\mathrm{mg} / \mathrm{kg})\end{array}$ & $\begin{array}{l}\text { Ex. } \\
\text { Acidity } \\
\text { (mg/l) }\end{array}$ & $\begin{array}{l}\text { Ext. } \\
\text { Alkalinity } \\
(\mathrm{mg} / \mathrm{l})\end{array}$ & $\begin{array}{l}\text { Mn } \\
(\mathrm{mg} / \mathrm{l})\end{array}$ & $\begin{array}{l}\text { Fe } \\
(\mathrm{mg} / \mathrm{kg})\end{array}$ & $\begin{array}{l}\mathrm{Cu} \\
(\mathrm{mg} / \mathrm{kg})\end{array}$ & $\begin{array}{l}\text { Zn } \\
(\mathrm{mg} / \mathrm{l})\end{array}$ & $\begin{array}{l}\mathrm{K} \\
(\mathrm{mg} / \mathrm{l})\end{array}$ & $\begin{array}{l}\mathrm{Ca} \\
(\mathrm{cmol} / \mathrm{kg})\end{array}$ & $\begin{array}{l}\text { Mg } \\
(\mathrm{cmol} / \mathrm{l})\end{array}$ & $\begin{array}{l}\mathrm{Na} \\
(\mathrm{cmol} / \mathrm{kg})\end{array}$ \\
\hline $0-15$ & $6.25 \pm 0.50$ & $28.03 \pm 0.65$ & $2.90 \pm 0.07$ & $37.11 \pm 1.54$ & $0.25 \pm 0.12$ & $1.53 \pm 0.17$ & $39.08 \pm 9.57$ & $39.53 \pm 15.58$ & $8.53 \pm 0.32$ & $9.01 \pm 0.66$ & $1.24 \pm 1.22$ & $11.02 \pm 0.38$ & $0.10 \pm 0.29$ & $2.95 \pm 0.08$ \\
\hline $15-30$ & $6.28 \pm 0.08$ & $23.5 \pm 2.01$ & $2.43 \pm 0.21$ & $35.43 \pm 0.69$ & $0.15 \pm 0.03$ & $1.20 \pm 0.12$ & $32.43 \pm 11.93$ & $51.83 \pm 14.62$ & $8.26 \pm 0.29$ & $6.62 \pm 0.21$ & $0.66 \pm 0.46$ & $10.85 \pm 1.05$ & $0.69 \pm 0.16$ & $2.90 \pm 0.09$ \\
\hline $30-45$ & $6.43 \pm 0.05$ & $19.24 \pm 1.43$ & $1.99 \pm 0.15$ & $35.06 \pm 0.65$ & $0.20 \pm 0.07$ & $1.13 \pm 0.11$ & $18.78 \pm 4.80$ & $34.33 \pm 10.68$ & $7.99 \pm 0.06$ & $4.42 \pm 1.11$ & $0.21 \pm 0.01$ & $10.42 \pm 1.29$ & $0.81 \pm 0.14$ & $2.97 \pm 0.06$ \\
\hline
\end{tabular}

Source: Fieldwork 
Journal DOI: www.doi.org/10.46654/ij.24889849 Vol. 7, Issue 2 (February, 2021) |www.ijaar.org

Article DOI: www.doi.org/10.46654/ij.24889849.e7218

Table 9: Heavy Metal Distribution of the Soils at AC, Ibadan

\begin{tabular}{|c|c|c|c|c|c|c|}
\hline Location & Depth (cm) & $\mathrm{Pb}$ (mg/kg) & $\mathrm{Cd}(\mathrm{mg} / \mathrm{kg})$ & Co (mg/kg) & $\mathrm{Cr}(\mathrm{mg} / \mathrm{kg})$ & $\mathrm{Ni}(\mathrm{mg} / \mathrm{kg})$ \\
\hline \multirow{3}{*}{ Pit } & $0-15$ & 5.80 & 9.5 & 10.35 & 17.1 & 15.37 \\
\hline & $15-30$ & 4.93 & 9.45 & 7.8 & 7.15 & 14.71 \\
\hline & $30-45$ & 4. 41 & 5.85 & 2.85 & 16.95 & 6.06 \\
\hline \multirow{3}{*}{ Central } & $0-15$ & 5.15 & 11 & 10.65 & 18.35 & 16.69 \\
\hline & $15-30$ & 4.30 & 10.4 & 9.65 & 17.3 & 16.14 \\
\hline & $30-45$ & 3.11 & 8.9 & 5.5 & 7.25 & 15.36 \\
\hline \multirow{3}{*}{$\mathrm{E} / \mathrm{W}$} & $0-15$ & 8.13 & 11.6 & 14.05 & 9.75 & 13.80 \\
\hline & $15-30$ & 6.87 & 10.7 & 11.25 & 18.7 & 13.09 \\
\hline & $30-45$ & 5.42 & 9.2 & 2.3 & 11.25 & 3.50 \\
\hline \multirow{3}{*}{$\mathrm{N} / \mathrm{S}$} & $0-15$ & 6.19 & 11.85 & 18.05 & 7 & 14.10 \\
\hline & $15-30$ & 2.78 & 11.25 & 13.4 & 6.65 & 9.79 \\
\hline & $30-45$ & 1.92 & 11 & 6.8 & 1.85 & 5.59 \\
\hline
\end{tabular}

Source: Fieldwork

Table 10: Mean of Heavy Metal Distribution of the Soils at AC, Ibadan

\begin{tabular}{lccccc}
\hline $\begin{array}{l}\text { Depth } \\
(\mathbf{c m})\end{array}$ & $\mathbf{P b}(\mathbf{m g} / \mathbf{k g})$ & $\mathbf{C d}(\mathbf{m g} / \mathbf{k g})$ & $\mathbf{C o}(\mathbf{m g} / \mathbf{k g})$ & $\mathbf{C r}(\mathbf{m g} / \mathbf{k g})$ & $\mathbf{N i}(\mathbf{m g} / \mathbf{k g})$ \\
\hline $0-15$ & $5.66 \pm 0.55$ & $10.55 \pm 0.36$ & $13.58 \pm 4.16$ & $12.53 \pm 3.17$ & $12.53 \pm 2.34$ \\
$15-30$ & $4.58 \pm 0.68$ & $10.23 \pm 0.60$ & $9.98 \pm 2.57$ & $12.1 \pm 3.75$ & $12.34 \pm 2.19$ \\
$30-45$ & $4.52 \pm 1.35$ & $9.4 \pm 1.33$ & $9.76 \pm 1.00$ & $10.18 \pm 2.40$ & $11.17 \pm 3.55$ \\
\hline
\end{tabular}


Journal DOI: www.doi.org/10.46654/ij.24889849

Vol. 7, Issue 2 (February, 2021) |www.ijaar.org

Article DOI: www.doi.org/10.46654/ij.24889849.e7218

Table 11: $\quad$ Physical Properties of the Soils at AC, Ibadan

\begin{tabular}{|c|c|c|c|c|c|}
\hline Location & Depth (cm) & Clay (g/kg) & Silt (g/kg) & Sand (g/kg) & Textural Class \\
\hline \multirow{3}{*}{ Pit } & $0-15$ & 108 & 280 & 612 & Sandy Loam \\
\hline & $15-30$ & 148 & 180 & 672 & Sandy Loam \\
\hline & $30-45$ & 128 & 140 & 732 & Sandy Loam \\
\hline \multirow{3}{*}{ Central } & $0-15$ & 108 & 140 & 712 & Sandy Loam \\
\hline & $15-30$ & 208 & 180 & 652 & Sandy Clay Loam \\
\hline & $30-45$ & 248 & 140 & 592 & Sandy Clay Loam \\
\hline \multirow{3}{*}{$\mathrm{E} / \mathrm{W}$} & $0-15$ & 148 & 260 & 592 & Sandy Loam \\
\hline & $15-30$ & 268 & 180 & 552 & Sandy Clay Loam \\
\hline & $30-45$ & 288 & 180 & 532 & Sandy Clay Loam \\
\hline \multirow{3}{*}{$\mathrm{N} / \mathrm{S}$} & $0-15$ & 88 & 180 & 732 & Sandy Loam \\
\hline & $15-30$ & 68 & 200 & 732 & Sandy Loam \\
\hline & $30-45$ & 68 & 200 & 732 & Sandy Loam \\
\hline
\end{tabular}

Table 12: Mean of Soil Particle Size Distribution at AC, Ibadan

\begin{tabular}{llll}
\hline Depth (cm) & Clay $(\mathbf{g} / \mathbf{k g})$ & Silt (g/kg) & Sand (g/kg) \\
\hline $0-15$ & $113 \pm 12.58$ & $215 \pm 33.04$ & $662 \pm 35.12$ \\
$15-30$ & $173 \pm 42.72$ & $185 \pm 5.00$ & $652 \pm 37.42$ \\
$30-45$ & $183 \pm 5123$ & $165 \pm 15.00$ & $647 \pm 50.58$ \\
\hline
\end{tabular}

Source: Fieldwork 
Journal DOI: www.doi.org/10.46654/ij.24889849

\section{Conclusion}

The study concluded that industrial activities are impacting on the soil quality with special reference to nutrient and heavy metal contents within and around industrial facilities. Soil Chemical properties, heavy metal distribution, and particle size distribution provided a base for considering the level of impacts industrial facilities have on soil qualities of receiving environments. Four categories of activities that could have affected soil properties or entire soil bodies included low level of enforcement of laws, environmentally unfriendly production methods, discharge of waste (solid, liquid, gaseous) and direct dumping on soils. Attention needs to be given to sustainable land use, safe production methods, increased level of monitoring of industrial operations to ensure they comply with extant laws, policies, standards and regulations that protect environmental quality. 


\section{REFERENCES}

Adams, B. A. (2010). Effect of waste water on vegetable production. Journal of Agriculture and Social Research 10(1): 140-143.

Adedeji, O. B., Adeyemo O. K. and Oyedele. M. O. (2011). Heavy metals in snail and water samples from Alaro river in Oluyole industrial area of Ibadan south western Nigeria Journal of Applied Sciences in Environmental Sanitation, 6(2): 115-121.

Adedipe, N. O, Sridhar, M. K. C. and Baker. J. (2005). Waste Management, Processing, and Detoxification In: Ecosystems and Human Well-being: Synthesis. Island press, Washington DC, pp 313-333.

Adeoye, G. O. (2008). Comparative evaluation of Organo-Mineral Fertilizer (OMF) and Mineral Fertilizer (NPK) on yield and quality of maize (Zea mays (L) Moench), Nigerian Journal of Soil Science, 18:132-137.

Agdex, (2010). Liming acid soils. Agri facts. Practical information for Alberta's agriculture industry, $4 p p$

Aghabarati, A., Hosseini, S. A., Maralian, H. (2008). Heavy metal concentration of soil and Olive trees (Olea eurapoea L) in suburban areas of Tehran, Iran, Res.J. Environ. Sci., 2, 323-329

Aina, P. O., Lal R. and Taylor G. S. (1980). Relative susceptibility of some Nigerian soils to water erosion. Nigeria Journal of Soil Science 1:1-18

Aiyesanmi, A. F (2005). Assessment of heavy metals contamination of Robertkiri oil fields soils. Nigerian Journal of Soil Science 15: 42-46

Alberti, M. (2008). Advances in urban ecology: Integrating humans and ecological processes in urban ecosystems, Springer Science Business Media, Seattle.

Awomeso, J. A. (2009). Impact of industrial effluents on water, soils and plants in the Alakia industrial area of Ibadan, South West Nigeria. Toxicological and Environmental Chemistry 91(1): 5-15.

Aweto, A. O. (1994). The Soils and Agro- Environment of the Ibadan Area. Unpublished Monograph.

Bowers, John. (1997). Sustainability and Environmental Economics - An Alternative Text. England: Londman.

Brady, R. R and Well (2009). Nature and Properties of Soils. Pp 1-34

El-Nennah, M., Kobbia, T., Shehata, A., Gamal, L. (2006). Effect of irrigation loamy sand soil by sewage effluents on its contents of some nutrients and heavy metals. Plant soil, 65, 289-292

El-Hady, B. A. A. (2007). Effects of polluted and River Nile irrigation water on contents of heavy metals of some soils and plants. Res. J. Agri. And Biol. 3, 287- 294

FFD (2012). Fertilizer use and management practices for crops in Nigeria. Aduayi E. A, Chude V. O., Adebusuyi B. A., Olayiwola S. O. (eds). Federal Fertilizer Department (FFD). Federal Ministry of Agriculture and Rural Development, Abuja. 166pp.

Lin J., Wang W., Li Y., and Yang L., (2010). Heavy Metals in Soil and Crops of an Intensively Farmed Area: A case Study in Yucheng City, Shandong Province, China.

NESREA (2009): National Environmental Regulations for Food, Beverages and Tobacco Sector, S.I. No. 33.

Ogedengbe, K. and C. O. Akinbile. (2010). Comparative analysis of the impact of industrial and agricultural effluent on Ona Stream in Ibadan, Nigeria. New York Science Journal 3(7): 25-33. 
Ogunyemi, S., Bamgbose, O. O., and Awodoyin. R. O. (2003). Heavy Metals contamination of some leafy vegetables growing within Ibadan Metropolis, South-western Nigeria. Tropical Agricultural Research and Extensions, 6:71-76.

Osibanjo, O., Daso A. P., and Gbadebo. A. M. (2011). The impact of industries on surface water quality of River Ona and River Alaro in Oluyole Industrial Estate, Ibadan, Nigeria. African Journal of Biotechnology 10 (4):696-702.

Smyth C. J. and Montgomery, R. F (1962): Soils and Land use in Central Western Nigeria, Government Printer, Ibadan.

Tiller, K. G. (1986). Essential and toxic heavy metals in soils and ecological relevance. Trans. Xiii congr. Intern. Soc. soil Sci. 1, 29-44

Zia M. H., Ahmad R., Khaliq I., Ahmad A and Irshad M. (2006). Micronutrients status and management in orchards soils: applied aspects. Soils and Environment 25(1): 6-16, 2006 